\title{
Secondary diabetes as a rare complication of glycogen storage disease 1a: case report and review of literature
}

Wtórna cukrzyca jako rzadkie powikłanie choroby spichrzeniowej glikogenu 1a: opis przypadku i przegląd literatury

\author{
Anshita Aggarwal, Deepika Patel, Bindu Kulshreshtha
}

Department of Endocrinology, ABVIMS \& Dr Ram Manohar Lohia Hospital, India

\begin{abstract}
Glycogen storage diseases (GSDs) are disorders of carbohydrate metabolism and hypoglycemia is their hallmark. Secondary diabetes with glycogen storage disease, which seems rather paradoxical, has been rarely reported. A 13-year-old girl previously diagnosed to have GSD 1a presented to the emergency with multiple episodes of vomiting and loss of consciousness. She had hyperglycemia, ketonuria, hyperlactatemia and metabolic acidosis, suggestive of diabetic ketosis with acidosis possibly contributed by both high serum lactate and serum ketones. Her glycated hemoglobin was high, with detectable serum insulin levels, which suggested secondary diabetes in the background of GSD la. Her management posed a therapeutic challenge. She was managed with insulin and achieved good glycemic control. We wish to conclude that GSD may rarely lead to secondary diabetes as a complication and the management is complex owing to the nature of the disease. Insulin remains the mainstay of the treatment.
\end{abstract}

Key words:

glycogen storage disease, diabetes, insulin.

\section{Introduction}

Glycogen storage diseases (GSDs) are rare, inherited disorders of carbohydrate metabolism. They are characterized by hypoglycemia, hepatomegaly, and many other clinical features and complications. The occurrence of secondary diabetes due to GSD sounds paradoxical and counter-intuitive but has been seen in anecdotal case reports. Various hypotheses for the pathophysiology have been suggested but the exact mechanism remains elusive. Herein we present a case of a young girl with GSD who presented with diabetic ketosis. The case posed a therapeutic challenge but was managed well with insulin.

\section{Case report}

A 13-year-old girl presented to the emergency department of our hospital with a history of loss of consciousness around an hour prior to presentation. There was a preceding history of multiple episodes of vomiting and reduced oral intake over the previous 2 days. There was no preceding history of headache, fever, head trauma, seizure, or abdominal pain. The metabolic screen of the patient revealed a blood glucose value of $300 \mathrm{mg} / \mathrm{dl}$ and hyperlactatemia (serum lactate - $3.8 \mathrm{mmol} / \mathrm{l}$ [normal $<2 \mathrm{mmol} / \mathrm{l}])$. The arterial blood gas analysis was suggestive of metabolic acidosis. The urine analysis was suggestive of ketonuria, which, coupled with hyperglycemia established the diagnosis of diabetic ketosis. It is likely that both the ketosis and the hyperlactatemia contributed to her metabolic acidosis. Her lipid profile and uric acid levels were normal. The patient was successfully managed with intravenous fluids and intravenous insulin infusion. Once her clinical condition stabilized, she was switched to sub-cutaneous premixed insulin. Further investigations revealed glycated hemoglobin $\left(\mathrm{HbA}_{1 \mathrm{c}}\right)$ of $11.5 \%$ and fasting serum insulin value of $2.1 \mu \mathrm{lU} / \mathrm{ml}$, thus confirming this episode as a presentation of type 2/secondary diabetes mellitus. She also had short stature (height SDS: -2.5 ), body mass index (BMI - $15 \mathrm{~kg} / \mathrm{mtsq}$ [-1.8 SDS]), transaminitis (aspartate aminotransferase $-95 \mathrm{U} / \mathrm{l}$ [normal $<40 \mathrm{U} / \mathrm{l}]$, alanine aminotransferase - $121 \mathrm{U} / \mathrm{l}$ [normal < $40 \mathrm{U} / \mathrm{l}]$ ), coarsened liver echotexture on ultrasonography, and advanced hepatic fibrosis on fibroscan. A review of the patient's past medical history and hospital records showed that her parents had been first cousins and that she had had multiple episodes of generalized tonic-clonic seizures 5-6 years back, which on evaluation were found to be secondary to severe hypoglycemia. These episodes usually occurred late in the night, at around $3 \mathrm{am}$, 
and were followed by a brief period of loss of consciousness. Thereafter, spontaneous recovery occurred by the early hours of morning. She also had a history of gradual distension of the abdomen ever since the age of 2 years, which was found to be due to hepatomegaly. An ultrasound which had been at 8 years of age had showed liver length of $11.7 \mathrm{~cm}$ and spleen length of $8.1 \mathrm{~cm}$, both of which were more than the mean for Indian children her age $(10.9 \mathrm{~cm}$ and $7.6 \mathrm{~cm}$, respectively). A possibility of glycogen storage disease (GSD) had been considered keeping in mind hypoglycemia and hepatomegaly. A liver biopsy was done for the same, which showed the presence of enlarged hepatocytes laden with abundant glycogen (periodic acid-Schiff positive) on histopathological examination, thus confirming the diagnosis of GSD. In view of her clinical presentation, her GSD was subtyped as GSD 1a, though a genetic confirmation could not be obtained. She was advised to have a meal consisting of uncooked corn starch (30 grams) at bedtime, following which her hypoglycemic episodes reduced drastically, and eventually subsided completely. She was doing well for the last 5 years to date when she presented with diabetic ketosis. She did not have any features to suggest myopathy or cardiac involvement. She had not attained menarche yet and had average scholastic performance. She had some records of high blood glucose over the last 1 year prior to presentation but she did not seek treatment for it. However, there was no history of weight loss, polydipsia or polyuria. She did not have family history of diabetes.

During the subsequent follow up, she had brittle control of diabetes, marred by wide fluctuations in blood glucose levels. Owing to this, she was switched to basal-bolus regimen of insulin from pre-mixed insulin and was taught the nuances of self-titration. She was advised to continue bedtime corn starch to minimize the risk of hypoglycemia. Following this, she gradually achieved satisfactory glycemic control $\left(\mathrm{HbA}_{1 \mathrm{c}} 7 \%\right)$ with infrequent episodes of hypoglycemia.

\section{Discussion}

Glycogen storage diseases are a group of rare diseases characterized by an inborn disorder of carbohydrate metabolism with autosomal recessive inheritance. Hypoglycemia is the hallmark of GSDs. They are divided into 8 sub-types depending on their influence on blood glucose levels, hepatic function, and muscle function. In normal physiology, in the fed state, the excess glucose is stored in the form of glycogen in the liver and the muscles, and this glycogen later breaks down into glucose (glycogenolysis) during the fasting state. This is achieved by the synchronized function of a group of enzymes, and the deficiency of any of these enzymes can lead to failure of glycogenolysis, leading to GSD. Glycogen storage disease 1a (Von Gierke disease) is caused due to deficiency of the enzyme glucose-6-phosphatase which is involved in the terminal step of glycogenolysis. It is characterized by severe hypoglycemia in early childhood, hyperlactatemia, hypertriglyceridemia, and hepatic and renal dysfunction due to excessive glycogen [glucose-6-phosphate (G6P)] deposition. There is no specific treatment for it. Bedtime corn starch meal or continuous overnight gastric feeding to prevent hypoglycemia remain the mainstay of treatment. It is seen that as age advances, the episodes of hypoglycemia become less frequent and may even disappear completely in many patients. This phenomenon is seen as a part of an adaptive response, wherein there is gradual down-regulation of pancreatic -cell GLUT-2 receptors, preventing glucose entry into the beta cells which is a pre-requisite for insulin secretion [1]. This eventually leads to an insulinopenic state but progression to frank diabetes has rarely been reported. The previous case studies which have reported diabetes in GSD have been summarised in Table I [2-8]. It is noteworthy that barring one case [2] none of these cases presented with diabetic ketosis unlike ours. Two of these previously reported patients developed diabetes as a complication of liver transplant [7] and 1 due to chronic pancreatitis [5]. Five of the remaining cases had secondary diabetes due to GSD.

Apart from insulinopenia, the other mechanism which has been postulated involves hepatic steatosis. The accumulation of G6P in the liver triggers de novo lipogenesis leading to fatty liver which in turn leads to insulin resistance [9]. The co-existence of hypertriglyceridemia and obesity, which are commonly seen in GSD1a, further adds fuel to the fire and can worsen insulin resistance. In one of the previously reported patients, hypertriglyceridemia leading to recurrent episodes of pancreatitis and pancreatogenous diabetes has been seen [5]. G6P is known to stimulate the enzyme 11 -hydroxysteroid dehydrogenase type 1 (11 HSD1) which increases cortisol production and contributes to the pathogenesis of the metabolic syndrome [10]. Owing to the rarity of the condition, no specific guidelines exist for the treatment of secondary diabetes in GSD. The presence of hepatic and/or renal dysfunction makes insulin an attractive choice but it may be a double-edged sword as these individuals are already prone to hypoglycemia. Metformin and SGLT-2 inhibitors are better avoided due to the theoretical risk of precipitating metabolic acidosis. Alpha-glucosidase inhibitors have been used earlier, particularly if hyperglycemia is predominantly post-prandial. Sulfonylureas and DPP-4 inhibitors may have a limited role in the background of insulinopenia and have not been tried. In the index patient, owing to severe hyperglycemia and hepatic dysfunction, insulin was considered for treatment with watchful monitoring for hypoglycemia and continuation of bedtime corn starch meal. Since basalbolus insulin regimen is the most physiological regimen, it was thought to be the most appropriate for the patient. Initially the control was brittle with frequent episodes of hyperglycemia and hypoglycemia. However, with careful monitoring and after teaching the patient the nuances of self-titration on insulin, the glycemic control improved to a great extent. At the initial presentation, the daily insulin requirement of the patient was very high, possibly owing to glucotoxicity $(1 \mu / \mathrm{kg} /$ day). Once stable glucose levels were achieved, the requirement came down to $0.5 \mu / \mathrm{kg} /$ day.

The patient continues to do well on this regimen with good glycemic control and very rare episodes of hypoglycemia. It is noteworthy that glucagon administration has no role in exoge- 


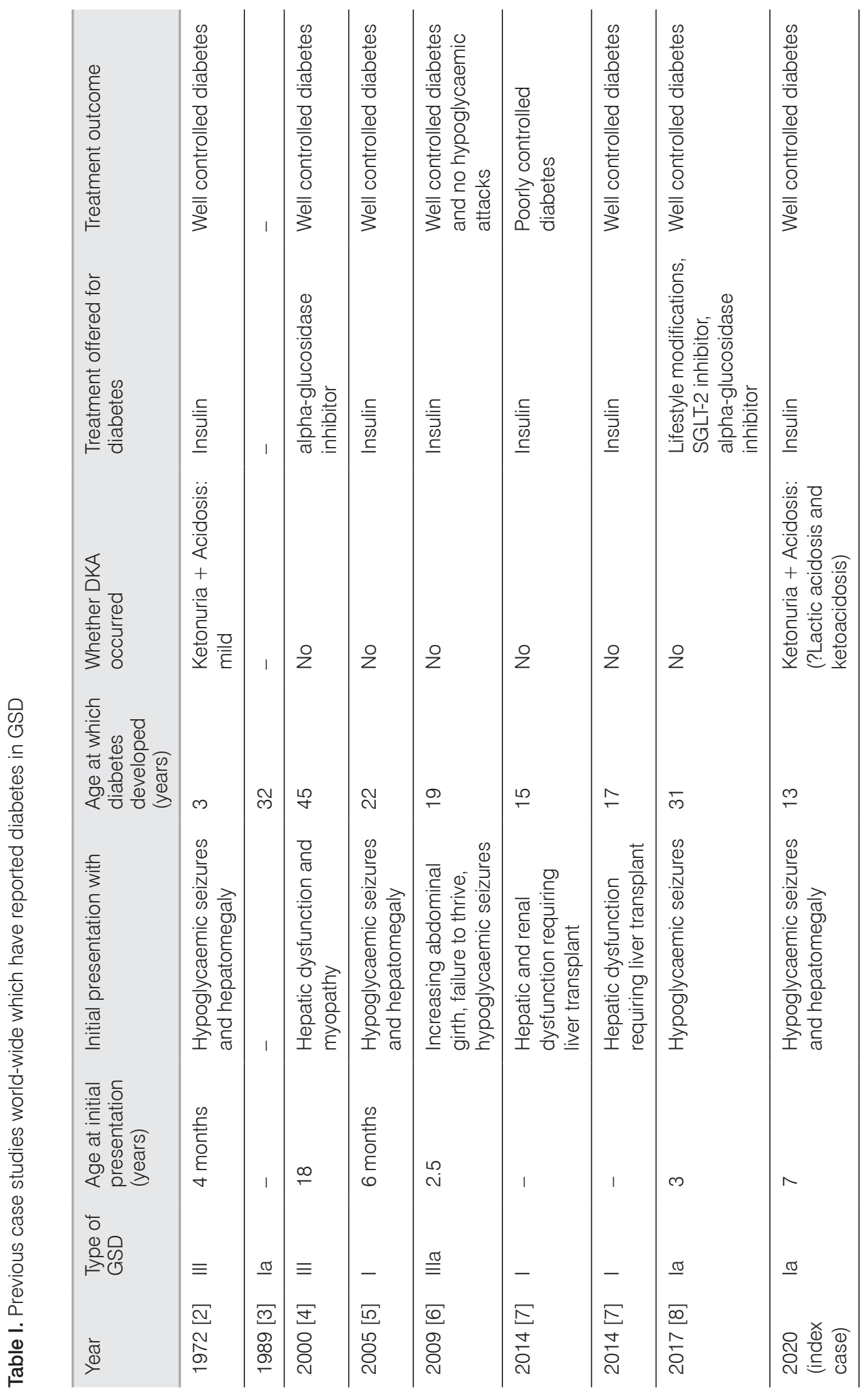


nous insulin-induced hypoglycemia in such patients and therefore must be avoided. It may rather precipitate lactic acidosis and cause decompensation.

\section{Limitations}

C-peptide levels and antibody levels could not be estimated owing to financial constraints. We accept that these are the limitations in our case. However, we believe that the presence of detectable serum insulin levels at the initial presentation goes against the possibility of type 1 diabetes.

\section{References}

1. Robinson S, Kessling A. Diabetes secondary to genetic disorders. Baillieres Clin Endocrinol Metab 1992; 6: 867-898. doi: 10.1016/ s0950-351x(05)80171-5.

2. Moe PJ, Waaler PE, Garatun Tjeldst $\varnothing$ O. Glycogen Storage Disease Type lii and Diabetes Mellitus. Acta Paediatrica 1972; 61: 483-486. doi: 10.1016/s0950-351x(05)80171-5.

3. Tanigawa K, Furuya H, Sato T, et al. A case of glycogen storage disease complicated by diabetes mellitus. Nippon Naika Gakkai Zasshi. 1989 Jun;78: 852-853. doi: 10.2169/naika.78.852.

4. Oki Y, Okubo M, Tanaka S, et al. Diabetes mellitus secondary to glycogen storage disease type III. Diabet Med 2000; 17: 810-812. doi: 10.1046/j.1464-5491.2000.00378.x.

5. Spiegel R, Rakover-Tenenbaum Y, Mandel H, et al. Secondary diabetes mellitus: late complication of glycogen storage disease type $1 \mathrm{~b}$. J Pediatr Endocrinol Metab 2005; 18: 617-619. doi: 10.1515/ jpem.2005.18.6.617.

\section{Conclusions}

Secondary diabetes may rarely be seen as a complication of GSD, and various pathophysiological mechanisms have been postulated for it. Owing to the presence of insulinopenia and the risk of lactic acidosis, the role of oral anti-diabetic agents is limited. Insulin is the mainstay of therapy but watchful monitoring is necessary, owing to the heightened risk of hypoglycemia in GSD.

6. Ismail H. Glycogen storage disease type III presenting with secondary diabetes and managed with insulin: a case report. Cases J 2009; 2: 6891. doi: 10.4076/1757-1627-2-6891.

7. Kumar K, Sachdev P, Randell T, Denvir L. Diabetes Mellitus a Late Complication in Glycogen Storage Disease Type 1b. Poster session presented at: $53^{\text {rd }}$ Annual Meeting of the European Society for Paediatric Endocrinology; 2014 Sept 20-22; Dublin, Ireland.

8. Cohn A, Ohri A. Diabetes mellitus in a patient with glycogen storage disease type la: a case report. J Med Case Rep 2017; 11: 319. doi: 10.1186/s13256-017-1462-5.

9. Rajas F, Labrune P, Mithieux G. Glycogen storage disease type 1 and diabetes: learning by comparing and contrasting the two disorders. Diabetes Metab 2013; 39: 377-387. doi: 10.1016/j.diabet.2013.03.002.

10. Czegle I, Csala M, Mandl J, et al. G6PT-H6PDH-11 HSD1 triad in the liver and its implication in the pathomechanism of the metabolic syndrome. World J Hepatol 2012; 4: 129-138. doi: 10.4254/wjh. v4.i4.129. 